\title{
А.А. ПЕчЕНКИН
}

\section{Квантовая логика и теория вероятностей ${ }^{1}$}

\author{
Печенкин Александр Александрович \\ Кафедра философии и методологии науки, \\ философский факультет, МГУ имени М. В. Ломоносова \\ 119991, Москва, ГСП-1, Ломоносовский проспект, д. 27, корп. 4 \\ E-mail: a_pechenk@yahoo.com
}

В статье дается обзор тех работ по квантовой логике, которые непосредственно связаны с математическим обоснованием квантовой механики, а именно - c формулированием квантово-теоретической теории вероятностей. Развитие математического аппарата квантовой механики связывается с линией: П.А.М. Дирак (его книга 1927 г. «Принципы квантовой механики»), И. фон Нейман («Математические основания квантовой механики» - 1932 г.), статья Г. Биркгофа и И. фон Неймана о квантовой логике (1936 г.). При этом показано, что дальнейшее развитие идей Биркгофа и фон Неймана привело к формулированию квантовотеоретической концепции вероятности, обобщающей классическую аксиоматику А.Н. Колмогорова и заполняющей те пробелы математической строгости, которые оставались в книге фон Неймана (1932 г.).

Ключевые слова: решетка, булева алгебра, частотное понятие вероятности, сигмаалгебра, наблюдаемая, состояние, концептуальное обоснование, математическое обоснование

\section{Введение}

$\mathrm{K}$ настоящему времени под рубрику «квантовая логика» подпадает большое число как математических, так и философских (относящихся к философии науки) публикаций (книг и статей). В настоящей статье рассматриваются те из них, которые оказались задействованы в развитии квантовой теории вероятностей и тем самым те, которые относятся к непосредственному совершенствованию математического аппарата квантовой механики. В книге фон Неймана «Математические основы квантовой механики» (1932 г.) понятие вероятности вводилось не на базе аксиоматики А.Н. Колмогорова, сложившейся

\footnotetext{
${ }^{1}$ Исследование выполнено при финансовой поддержки РФФИ. Проект № 1533-0141.
}

(C) Печенкин А.А. 
в начале 1930-х гг., а в русле идей Р. фон Мизеса, в русле его частотной концепции вероятности. В то же время, как отмечает Редеи, фон Нейман был неудовлетворен концепцией «вероятность - предел последовательности относительных частот», развитой фон Мизесом в 1920-е гг. Он стремился к логически последовательному изложению квантовой механики, к изложению, в котором теория вероятностей не была бы дополнением к логически строгой квантовой теории, а была бы органически вписана в это изложение.

Какую же роль в этом изложении квантовой механики сыграла квантовая логика? Как признано в большинстве публикаций, начало квантовой логики было положено статьей Г. Биркгофа и И. фон Неймана 1936 г. В ней было развито исчисление высказываний, которое, в отличие от классического, не выполнялось правило дистрибутивности. Биркгоф и фон Нейман показали, что квантовомеханические высказывания образуют решетку (lattice), алгебраический объект, обобщающий понятие булевой алгебры (в российской литературе раньше, в 60-70-е гг. чаще использовался термин «структура» $)^{2}$. Причем решетка квантово-механических высказываний оказывается недистрибутивной, небулевой. Однако эта решетка является ортомодулярной: ортомодулярной называют решетку, которая одновременно является ортодополнительной и модулярной. Ортодополнительной называют решетку с нулем и единицей, для которой для любого элемента а существует элемент $a^{\perp}$, отвечающий свойству $a \vee a^{\perp}=1$, свойству $a \& a^{\perp}=0$ и ряду других свойств $[1,10,12]$. Модулярной называют решетку, в которой при условии $a \leq c(\leq-$ отношение порядка) выполняется а $\vee(b \& c)=(a \vee b) \& c$.

Статья Биркгофа и фон Неймана индуцировала обширные изыскания в логике. Далеко не все из них были связаны с разработкой идеи квантово-теоретической вероятности. Многие исследования по квантовой логике оказались опытами построения неклассических логик (модальных, временных, многозначных...). Статья

\footnotetext{
${ }^{2}$ Решетка - это частично упорядоченное множество, в котором вводятся понятия $\sup (a, b)$ и inf $(a, b)$ - наименьшей верхней грани и наибольшей нижней грани. Если речь идет о множестве всех подмножеств, упорядоченных по включению, то sup есть объединение подмножеств, а inf есть их пересечение.
} 
же Биркгофа и фон Неймана была не просто опытом в области логики, эта статья оказалась шагом в развитии концептуальных оснований квантовой механики: к ней восходит квантово-теоретическая концепция вероятности. Классическая теория вероятностей Колмогорова была построена на базе алгебраической структуры, называемой сигма-алгеброй. Сигма-алгебра - это теоретико-множественная структура, отвечающая отношению дистрибутивности, являющаяся булевой. Квантовая логика - небулева. Однако свойство ортомодулярности позволяет построить булевые подрешетки и определить на них вероятностную меру.

В следующем параграфе будет прослежена эволюция взглядов фон Неймана относительно квантово-теоретической вероятности. Этот параграф покажет те логические трудности в изложении квантовой механики, которые привели к построению обобщенной теории вероятностей. Затем (второй параграф) будет описана структура неклассической квантово-теоретической вероятности. В третьем параграфе будет представлена концепция математического обоснования квантовой механики. Математическое обоснование будет рассмотрено как концептуальной обоснование, направленное на выяснение предельных предпосылок теории. В этой связи обобщенная квантово-теоретическая вероятность будет охарактеризована как этап в математическом обосновании квантовой механики.

\section{1. Эволюция взглядов фон Неймана на квантово-теоретическую вероятность}

В книге 1932 г. фон Нейман исходил из частотной теории вероятности фон Мизеса: вероятность трактовалась как предел последовательности относительных частот. Позиция фон Неймана здесь была традиционной. Как известно, в 1936 г. М. Борн предложил статистическую интерпретацию волновой функции (еще раньше вероятностные представления были введены в старую квантовую теорию БораЗоммерфельда и в матричную механику). Первоначально логические и философские трудности, связанные со статистической интерпретацией казались менее важными, чем экспериментальные факты, свидетельствующие в ее пользу. Лишь открытие соотношения 
неопределенностей заставило с интересом отнестись к этим трудностям. Однако первые рефлексии по поводу теоретико-вероятностных представлений носили лишь интуитивный характер. Физики еще не ставили задачу синтеза вероятностных идей и математического аппарата квантовой механики. Они молчаливо принимали, что в квантовой механике используется классическая теория вероятностей, и пытались осмыслить возникающие при этом аномалии путем новых интерпретаций. Так, В. Гейзеберг связывал вероятность, возникающую в квантовой механике, с тенденцией (потенцией в смысле аристотелевской философии) и, в отличие от вероятности в классической физике, считал квантовую вероятность полностью объективной [3, с. 32].

Существенный шаг к строгой трактовке вероятности в квантовой механике сделал фон Нейман в книге 1932 г. Фон Нейман сформулировал статистический алгоритм, соотносящий математический формализм и результаты измерений, который включал все статистические утверждения, делавшиеся раньше. Этот алгоритм состоял из двух положений: 1) вероятность того, что наблюдаемая $R$ в состоянии $\phi$ принимает значение из интервала $I$, равна $|E(I) \phi|^{2}$, где $\mathrm{E}(I)-$ разложение единицы, принадлежащее наблюдаемой $R, 2)$ математическое ожидание $R$ в состоянии $\phi$ равно скалярному произведению $(R \phi, \phi)$.

Однако у фон Неймана понятие вероятности оставалось внешним по отношению к основной математической схеме квантовой механики - теории самосопряженных операторов в гильбертовых пространствах. У него еще нет обобщенной теории вероятностей, преодолевающей ограниченности той трактовки, которая была выдвинута фон Мизесом.

Как отмечает М. Редеи, принятая фон Нейманом в 1932 г. интерпретация вероятности как относительной частоты вела к концептуальным трудностям. Одна из этих трудностей - понятие ансамбля и понятие отбора подансамбля путем квантового измерения [14]. В 1932 г. фон Нейман еще полагает, что можно сохранить ансамблевую интерпретацию, оставляя в стороне проблему квантового возмущения этого ансамбля в ходе измерения. 
Фон Нейман писал следующее:

Даже если две или более величины $R, S$ не могут быть одновременно измеряемы, по отношению к единичной системе, их вероятностные распределения в данном ансамбле $\left\{S_{1}, S_{2}, \ldots, S_{N}\right\}$ могут быть получены с произвольной точностью, если $N$ достаточно велико. Действительно, в случае ансамбля из $N$ элементов достаточно собрать статистические показания о распределении значений величины $R$ не со всех $N$ элементов $S_{1}, S_{2}, \ldots, S_{N}$, а лишь с некоторой подсистемы из $M$ элементов, где $M$ меньше $N$, скажем, $S_{1}, S_{2}, \ldots, S_{M}$, если только $M$ и $N$ достаточно велики, причем $M$ можно сделать совсем малым по сравнению с $N$. Тогда при измерении будет подвергнута изменению только $M / N$ я часть ансамбля, сколь угодно малая. Чтобы измерить одновременно две величины, скажем, $R, S$, нам потребуются две подсистемы, скажем, $\left\{S_{1}, S_{2}, \ldots, S_{M}\right\}$ и $\left\{S_{M+1}, \ldots, S_{2 M}\right\}$, так что первая будет применена для снимка статистики $R$, а вторая - для снимка статистики $S$. Тогда оба измерения не помешают одно другому - хотя и производятся на одном и том же ансамбле - и даже изменят этот ансамбль на произвольно малую величину [6, с. 254].

Как замечает Редеи, в основе приведенного рассуждения просматривается допущение, что субансамбли представляют большой ансамбль в том смысле, что относительная частота любого свойства будет той же самой как в подансамбле, так и первоначальном ансамбле. Это нетривиальное допущение, известное как требование иррегулярности, принимается в отношении ансамблей, применяемых при расчете вероятности как относительной частоты (см., например, [4, c. 47]). Данное допущение, пишет Редеи, уже критиковалось при изложении вероятности по фон Мизесу [14, p. 156].

В 1937 г., как констатирует Редеи, фон Нейман в статье «Квантовая логика: строгая и вероятностная логика» отказался от безусловной поддержки частотного понятия вероятности, выдвинутого фон Мизесом [14, p. 166].

\section{2. Понятие решетки и обобщенная теория вероятностей}

Классическое понятие вероятности строится на базе алгебраической структуры, называемой сигма-алгеброй. Но фон Нейман приходит к 
небулевой решетке, в то время как сигма-алгебра, задействованная у А.Н. Колмогорова, является булевой решеткой ${ }^{3}$. Речь идет, следовательно, о построении обобщенной теории вероятностей.

Рассмотрим логическое исчисление проекторов. Что такое проектор (оператор проектирования)? Согласно фон Нейману, состояние системы, которое, согласно волновой механике Шредингера, выражено волновой функцией, представляет вектор гильбертова пространства. Наблюдаемая (координата, импульс, спин и т. д.) - это самосопряженный оператор, действующий в гильбертовом пространстве (мы опускаем более точную формулировку). Фон Нейман, кроме наблюдаемых, использует представление об альтернативных свойствах физических систем (например, это следующие свойства: иметь спин +1 , иметь импульс 2 кгм/сек, электрон может иметь спин +1 или не иметь такого спина - иметь спин -1--, иметь импульс 2 кгм/сек или не иметь такового). Свойства представляют особые наблюдаемые проекционные операторы (проекторы). Значение проектора - это 0 или 1. Если $P$ - проекционный оператор, то $P^{2}=P$ и, наоборот, если $P^{2}=P$, то $P$ - проекционный оператор.

Проекторы находятся во взаимно-однозначном соответствии с замкнутыми подпространствами гильбертова пространства. Действительно, если $P$ - проекционный оператор, его ранг (область определения) есть замкнутое множество, и любое замкнутое подпространство составляет ранг одного проектора.

Логическое исчисление проекторов обнаруживает неклассическую логику. «Мы видим, что связь между свойствами физической системы, с одной стороны, и проекционными операторами, с другой стороны, - писал фон Нейман в своей книге 1932 г., - делает возможным некое логическое исчисление над ними. Однако в противоположность исчислению обычной логики эта система обогаще-

\footnotetext{
${ }^{3}$ А.Н. Колмогоров (1936) исходит из множества $U$ элементарных событий. Далее рассматривается некоторая система $F$ подмножеств множества $U$. Если подмножества $A_{1}, A_{2}, \ldots, A_{n}$ множества $U$ являются элементами множества $F$, то их сумма и произведение также являются элементами $F$. Множество $F$ называется $\sigma$-алгеброй событий.
} 
на характерным для квантовой механики понятием "одновременной рассудимости"» [6, с. 189].

В статье Биркгофа и фон Неймана это логическое исчисление было эксплицировано. Упорядоченное посредством теоретикомножественного включения замкнутые подпространства гильбертова пространства $H$ образуют полную решетку, в которой конъюнкция (наибольшая нижняя грань) множества подпространств есть их пересечение, тогда как дизъюнкция (наименьшая верхняя грань) есть их замкнутое объединение.

Исчисление Биркгофа-фон Неймана было небулевым. Этим была выражена его неклассичность. С этим исчислением не может связано классическое колмогоровское понятие вероятности. Однако, как показывают исследования последнего времени, понятие вероятности может быть обобщено и применено к логике проекционных операторов.

Вот что говорится об этом в одной из последних работ [16]. «Поскольку типичное замкнутое подпространство имеет бесконечно много дополнительных подпространств, эта решетка недистрибутивна, однако она ортодополнительна исходя из отображения

$\mathrm{M} \rightarrow M^{\perp}=[v \in H: \forall u \in M(v, u)=0]$.

Исходя из упомянутого взаимно однозначного соответствия между замкнутыми подпространствами и проекторами, мы можем ввести на множестве $L(H)$ структуру полной ортодополнительной решетки, определяя $P<Q$, если $\operatorname{ran}(P)<\operatorname{ran}(Q)$ и $P^{\prime}=1-P$ (так что $\left.\operatorname{ran}\left(P^{\prime}\right)=\operatorname{ran}(P)^{\perp}\right)$. Это есть непосредственно то, что $\mathrm{P} \leq Q$ только тогда, когда $P Q=Q P=P$. Обобщая, мы можем утверждать, что если $P Q=Q P$, то $P Q=P \wedge Q$, пересечение $P$ и $Q$ в $L(H)$. В этом случае их объединение дается как $P \vee Q=P+Q-P Q$.

Если $P$ и $Q$ проекционные операторы в гильбертовом пространстве, то следующие утверждения эквивалентны:

1) $P Q=Q P$

2) Подрешетка $L(H)$ генерированная $P, Q, P^{\prime}$ и $Q^{\prime}$ булева.

3) $P, Q$ лежат в общей булевой ортогональной подрешетке. 
Принимая идею, что коммутирующие наблюдаемые, в частности проекционные операторы, одновременно измеримы, мы заключаем, что элементы булевой ортогональной подрешетки $L(H)$ одновременно измеримы. Это значит, что мы можем сохранить классическую логическую интерпретацию пересечения, объединения и ортогонального дополнения в применении к коммутирующим проекционным операторам».

Переходим непосредственно к определению вероятности.

Назовем проекторы ортогональным и будем писать $P \perp Q$, если $P \leq Q^{\prime}$. Заметим, что $P \perp Q$, если $P Q=Q P=0$. Если $P$ и $Q$ ортогональные проекторы, то их объединение есть просто их сумма $\mathbf{P} \oplus \mathbf{Q}$. Тождественное отображение на $H$ будем обозначать как $\mathbf{1}^{\prime \prime}$.

ОПРЕДЕЛЕНИЕ. Счетно-аддитивная вероятностная мера на $L(H)$ представляет собой отображение $\mu: L \rightarrow[0,1]$ такое, что $\mu(1)=1$ и для любой последовательности ортогональных проекционных операторов $P_{i}, i=1,2, \ldots \mu\left({ }_{i} P_{i}\right)=\Sigma \mu\left(P_{i}\right)$ (вероятностная мера суммы равна сумме вероятностных мер).

Одним из достижений логико-алгебраического подхода явилось строгое доказательство теоремы Глиссона (1957 г.), показывающей (с современной точки зрения), что каждая счетно-аддитивная вероятностная мера на $L(H)$ имеет форму $\mu(P)=\operatorname{Tr}(W P)$, где $W$ оператор плотности, обобщающий понятие чистого состояния (вектор в гильбертовом пространстве) на смешанный случай. Чистый случай - это чистое состояние, выраженное волновой функцией или вектором в гильбертовым пространстве. Более общий смешанный случай представляет собой набор чистых состояний, имеющих свои «веса».

\section{3. Концептуальное обоснование квантовой механики у Дирака и фон Неймана}

Квантово-теоретическая трактовка вероятности явилась не только результатом кризиса частотной концепции вероятности, которой придерживался фон Нейман в его книге 1932 г. Эта трактовка восходит к общей идее концептуального обоснования квантовой механики, к общей идее последовательного и строгого изложения фи- 
зической теории. Как и многие другие теории, квантовая механика возникла в виде ряда вычислительных алгоритмов. Эти алгоритмы были связаны с наглядными схемами, моделями и философскими дискуссиями. Но в истории физики проглядывается и другая тенденция - тенденция к логически строгому изложению теории, к изложению на базе собственных принципов и концептуальных идей. Именно эта тенденция воплотилась в фоннеймановском изложении квантовой механики, в строгом изложении на базе последовательного и четкого построения математического аппарата этой теории. Эвристические соображения и наглядные модели при этом заключались в скобки, ставились в кавычки. Главное состояло в том, чтобы четко сформулировать исходные принципы квантовой механики и провести строгую математическую дедукцию тех положений, которые обеспечивают результативность этой теории.

Квантовая логика, теория ортодополнительных недистрибутивных решеток, изложенная Биркгофом и фон Нейманом, возникла в продолжение этих усилий. Квантовая логика позволила инкорпорировать идею вероятности, которая у фон Неймана в 1936 г. была дополнением к его изложению квантовой механики, непосредственно в структуру исходных положений квантовой этой теории.

В книге автора настоящей статьи под концептуальным обоснованием физической теории понимается выявление и уточнение ее исходных понятий и принципов. Критерием концептуальной обоснованности служит целостность образующейся теоретической системы. Исходными понятиями и принципами теории будут понятия и положения, обеспечивающие ее построение в соответствии с правилами дедуктивной логики и позволяющие осуществить интеграцию ее частных формулировок [7, с. 93].

При этом автором настоящей статьи был подчеркнут критический характер процедуры концептуального обоснования. Обоснование физической теории не сводится к математическому изложению этой теории. «В ходе концептуального обоснования критически пересматривается структура теории. Перед исследователем возникают вопросы: по праву то или иное положение оказалось исходным в структуре теории? Не является ли оно в действительности про- 
изводным и даже вспомогательным? Концептуальное обоснование предполагает также поиск логических ошибок в структуре теории. При этом речь не идет об ошибках, допущенных по недосмотру или из-за невежества. Такие ошибки вообще не рассматриваются в философии науки. В ходе концептуального обоснования выявляются логические ошибки, порожденные деформацией структуры теории, логические ошибки, незаметные при обычном некритическом подходе к теории» [7, с. 105].

Как известно, квантовая механика возникла в виде двух теорий - матричной механики и волновой. Проблема концептуального обоснования квантовой механики приобрела в 1926-27 гг. вид проблемы, названной М. Джеммером гиппарховой (по имени древнегреческого астронома Гиппарха, который во II в. до н. э. заинтересовался вопросом, почему одни и те же наблюдения одинаково хорошо укладываются в две различные теории движения небесных тел - в теорию концентрических окружностей с эпициклами и теорию эксцетрических окружностей [13, р. 307].

Суть гиппарховой проблемы в данном случае состояла в следующем. Матричная и волновая теории существенно различались и в плане математического аппарата, и в плане математических образов, выражающих физический смысл. Однако обе вели к одним и тем же предсказаниям эмпирических фактов. Решение гиппарховой проблемы было достигнуто на пути математического обоснования: матричная и волновая теории уступили место более совершенной формулировке, базирующейся на более совершенном математическом аппарате. Матричная и волновая теории не ушли из квантовой физики - они лишь приобрели служебный вспомогательный характер. Точнее, математические схемы этих теорий стали рассматриваться как представления, реализации математического формализма, а их наглядные образы были сведены на роль необязательных аналогий, поясняющих лишь некоторые аспекты физической ситуации.

Построение единой квантовой механики было достигнуто на пути развития квантовой теории преобразований, с которой, по словам Дирака, связана «сущность нового метода в теоретической физике» [5, с. 9]. Теория преобразований позволила абстрагироваться 
от тех черт квантовой механики, которые ассоциировались с историческими путями ее развития, индуцировались аналогиями с классической физикой, вытекали из требования наглядности, и тем самым позволила разглядеть в различных формулировках квантовой механики единый образ физической реальности. Правда, теория преобразований, которой было суждено объединить квантовую механику, первоначально формировалась с иными целями. Она формировалась в матричной механике в связи с решением конкретных задач, в связи с разработкой вычислительного алгоритма (Дирак, Иордан, Лондон, 1925-26 гг.). Когда в 1926 г. появилась волновая механика Шредингера, Лондон и Дирак занялись переносом методов теории преобразований, развитых в матричной механике, в волновую механику. В ходе этого переноса и произошло то «обращение метода», которое превратило теорию преобразований из результата квантовой механики в ее основание. Разработка теории преобразований оказалась связанной с выявлением математических структур, реализующихся как в квантовой, так и в волновой теории, а также с нахождением общего способа соотнесения математических выражений с результатами эксперимента. Как писал И. фон Нейман, матричная и волновая формулировки оказались сплавлены в единую теорию преобразований, где они дополнили друг друга и где оказалось возможным наиболее простое с математической точки зрения понимание физических вопросов.

Рассмотрим гиппархову проблему, возникшую в квантовой механике, подробнее. Матричная и волновая теории, охватывая по сути дела одну и ту же сферу опыта, различались в плане математического. В основе волновой механики лежало дифференциальное уравнение второго порядка, напоминающее уравнения механики жидкостей, уравнение Шредингера. Это уравнение формулировалось относительно $\psi$-функции, выражающей состояние физической системы и подчиняющейся принципу суперпозиции (как и состояния свободных полей). Шредингер в 1926 г. интерпретировал как некоторое непрерывное распределение электричества в реальном пространстве (плотность этого распределения считалась пропорциональной квадрату модуля $\psi$-функции). Правда, в том же 1926 г. М. Борн подверг 
интерпретацию Шредингера критике и предложил свое вероятностное истолкование $\psi$-функции. Но и у Борна она выступала как своего рода волна, волна амплитуды вероятности в конфигурационном пространстве.

Если математический аппарат волновой механики был аналитическим, то матричная механика опиралась на алгебру. Последняя исходила из наблюдаемой дискретности спектральных линий и выдвигала на первый план момент прерывности. Хотя матричная механика строилась на отрицании таких наглядных образов, как координата и циклическая частота электрона, наглядность, как отмечает М. Джеммер, в ней неявно присутствует.

Первым этапом в решении гиппарховой проблемы в квантовой теории оказалась статья Шредингера [8], в которой устанавливалась математическая эквивалентность матричной и волновой теорий. Эта эквивалентность уживалась у Шредингера с физической неэквивалентностью. Будучи привержен континуальным волновым представлениям, он считал более точным образом физической реальности волновую теорию. Матричная же механика была для него скорее формальной схемой.

Однако Шредингер установил не столько математическую эквивалентность, сколько, как отмечал Н.Р. Хэнсон, взаимопереводимость математических схем матричной и волновой формулировок [11]. У Шредингера не было общей математической схемы, по отношению к которой матричная и волновая теории выступали бы как представления, реализации. Он не увидел, что вектор-столбец матрицы преобразования подобия, применявшейся в матричной механике для решения задачи диагонализации матрицы, выражающей физическую величину (энергию), может быть отождествлен с его волновой функцией, а соответствующее матричное уравнение может быть преобразовано в его волновое уравнение. Это увидел позже Дирак, предложивший более общую математическую схему квантовой механики. Шредингер же просто показал, что «решение всей системы матричных уравнений Гейзенберга-Борна-Йордана сводится к естественной краевой задаче на собственные значения для некоторого линейного дифференциального уравнения в частных производ- 
ных» [8, с. 68-69], идентичного с волновым уравнением. Если эта задача решена, то оказывается возможным вычислить этот элемент «с помощью дифференцирований и квадратур».

Гиппархова проблема была решена Дираком, который отказался спорить о том, какая из схем (матричная или волновая) предпочтительнее. Дирак развивал свою концепцию квантовой механики в тесном контакте с работами по матричной теории и делал критические замечания в адрес работ по волновой механике. Тем не менее он подчеркивал дистанцию, отделяющую его от этого типа концептуальных построений. В своей итоговой книге (1930 г.) Дирак пишет о символическом методе, непосредственно оперирующем «в абстрактной форме фундаментальными величинами теории (инвариантами и квазиинвариантами преобразований)» и о методе представлений, который «оперирует системами чисел, соответствующих этим величинам» [5, с. 10]. Матричная и волновая теории строились методом представлений, Дирак же предпочитает символический метод, который «глубже проникает в природу вещей» и порывает с историческим способом изложения.

Дирак отклоняет наглядность как то требование, которому должна удовлетворять теория. «Согласно классической традиции, писал Дирак, - окружающий нас мир рассматривался как совокупность наблюдаемых объектов (частиц, флюидов, и т. д.), движущихся под действием сил согласно определенным законам, так что теория допускает наглядное представление в пространстве и времени. . Но в настоящее время становится все более очевидным, что природа действует иначе. Ее основные законы не управляют непосредственно миром наших наглядных представлений, но относятся к таким понятиям, о которых мы не можем составить себе наглядных представлений не впадая в противоречие» [5, с. 8].

Матричная и волновая теории трансформировались Дираком в частные формулировки квантовой механики, построенной при помощи символического метода и соотнесенной с результатами эксперимента посредством некоторого обобщения вероятностной интерпретации волновой функции, выдвинутой ранее М. Борном. Символический метод покоился на теории унитарных преобразований гильбер- 
това пространства. Матричная механика оказывается частной формулировкой квантовой механики в энергетическом представлении (в котором диагональна матрица энергии), формулировкой, использующей картину движения Гейзенберга, а волновая - формулировкой квантовой механики в координатном представлении, использующей картину движения Шредингера.

Фон Нейман уточнил дираковское обоснование квантовой механики, указав на скрытую зависимость построений Дирака от идеологии матричной механики (фон Нейман зафиксировал то, что Дирак находился под властью онтологии дискретного). Фон Нейман подчеркнул, что у Дирака, построившего квантовую механику как теорию самосопряженных операторов в гильбертовом пространстве, математически строго были изложены только проблемы дискретного спектра. Проблемы же непрерывного (сплошного) спектра у него не укладывались в гильбертово пространство. Чтобы достигнуть единообразия с трактовкой дискретного спектра, Дирак «лицемерно» (слова фон Неймана) допустил существование несобственных функций типа $\delta$-функции, для которых в то время не было строгой теории. Фон Нейман же изложил всю квантовую механику как теорию самосопряженных операторов в гильбертовом пространстве. «То, что не принадлежит $\mathbb{R}_{\infty}$ (бесконечно-мерному гильбертову пространству), для нас не существует» [6, с. 101]. Он разработал теорию самосопряженных операторов и построил единую теорию дискретного и непрерывного спектров. Он также сформулировал в общей форме правило, связывающее математический аппарат квантовой механики с экспериментом и наблюдением.

Следующий этап - логико-алгебраическое изложение центральных понятий квантовой механики, ее трактовка как обобщенной теории вероятностей.

\section{Заключение}

В настоящей статье построение теории квантовых высказываний Биркгофом и фон Нейманом и последующее развитие обобщенной теории вероятностей, базирующееся на этой работе Биркгофа и фон Неймана, трактуется как этап математического обоснования квантовой механики. 


\section{Литература}

[1] Васюков В.Л. Квантовая логика. М.: ПЕР СЭ, 2005. 192 с.

[2] Колмогоров А.Н. Основные понятия теории вероятностей. М.: ОНТИ, 1936. $120 \mathrm{c.}$

[3] Гейзенберг В. Физика и философия. М.: Наука, 1963. 464 с.

[4] Гнеденко В.Б. Курс теории вероятностей. М.: Наука, 1976. 448 с.

[5] Дирак П.А.М Принципы квантовой механики. М.: Наука, 1979. 278 с.

[6] фон Нейманн И. Математические основы квантовой механики. М.: Наука, 1964. 367 с.

[7] Печенкин A.A. Обоснование научной теории: классика и современность. М.: Наука, 1991, 184 с.

[8] Шредингер Э. Избранные труды по квантовой механике. М.: Мир, $1980,420 \mathrm{c}$.

[9] Birkhoff G., Neumann J. The Logic of Quantum Mechanics // Annals of Mathematics. 1936. Vol. 37. No. 4. P. 823-843.

[10] van Fraassen B.C. The labyrinth of quantum logics // Boston Studies in the Philosophy of Science / R.S. Cohen and M.W. Vastovsky (eds.). Dordrehct: Reidel Publishing Company, 1972. Vol. 13. P. 224-254.

[11] Hanson N.R. The concepr of positron. Cambridge: Cambr. Univ. Press, 1965. 236 p.

[12] Hooker $C$. The logico-algebraic approach to quantum mechanics. Dordrehct: Reidel Publishing Company, 1975. 469 p.

[13] Jammer $M$. The conceptual development of quantum mechanics. N. Y.: Wiley, 1966. 308 p.

[14] Redei M. John von Neumann on mathematical and axiomatic physics// The role of mathematics in physical sciences / Ed by G.Boniolo et. al. Netherlands: Springer, 2005. P. 43-52.

[15] Redei M. Von Neumann's concept of quantum logic and quantum probability // John von Neumann and the Foundations of Quantum Physics / Ed. by M. Redei, M. Stotzner. Netherlands: Springer, 2001. 464 p.

[16] Wilce A. Quantum logic and probability theory // Stanford encyclopedia of philosophy. 2002. Substantive revision - 2017. URL: https:/ plato.stanford.edu/entries/qt-quantlog (дата обращения: 30.08.2017). 


\title{
A.A. PeChenkin \\ Quantum Logic and Probability Theory ${ }^{1}$
}

\author{
Pechenkin Alexandr Alexandrovich \\ Department of philosophy and methodology of science, \\ Faculty of Philosophy, Lomonosov Moscow State University \\ Lomonosovsky prospect, 27-4, GSP-1, Moscow, 119991, Russian Federation. \\ E-mail: a_pechenk@yahoo.com
}

The paper provides the review of the texts on quantum logic, the texts which are directly connected with the mathematical foundations of quantum mechanics. These are texts which discuss the theory of quantum probability. The development of the mathematical scheme of quantum mechanics is discussed along the following line: P.A.M. Dirack's 1927 "The Principles of Quantum Mechanics", J. von Neumann's 1932 "Mathematische Grundlagen der Quantenmechanik", G.Birkgoff-I.von Neumann's 1936 "The logic of quantum mechanics". It is shown that the further development of the mathematical foundations of quantum mechanics resulted in the construction of quantum theory of probability, the theory generalizing A.N.Kolmogorov's classical probability and critically improving von Neumann's 1932 mathematical scheme.

Keywords: lattice, boolean algebra, $\sigma$-algebra, frequency understanding of probability, mathematical justification

\section{References}

[1] Vasukov, V.L. Kvantovaya logika [Quantum logic]. Moscow: Per Se, 2005. 192 pp. (In Russian)

[2] Kolmogorov, A.N. Osnovnie ponyatita teorii veroyatnostey [Foundations of the Theory of Probability]. Moscow: ONTI, 1936. 120 pp. (In Russian).

[3] Heisenberg, V. Fizika i filosofiya [Physics and Philosophy]. Moscow: Nauka, 1963. 464 pp. (In Russian)

[4] Gnedenko, V.B. Kurs teorii veroyatnostey [Course in Probability Theory]. Moscow: Nauka, 1976. 448 pp. (In Russian)

[5] Dirak, P.A.M. Principi kvantovoy mehaniki [The Analytic Hierarchy Process]. Moscow: Nauka, 1979. 278 pp. (In Russian)

\footnotetext{
${ }^{1}$ The paper is supported by Russian Foundation for Basic Research, projects № 15-33-0141.
} 
[6] Von Neumann, J. Matematicheskie osnovy kvantovoi mehaniki [Mathematical foundations of quantum mechanics]. Moscow. Nauka, 1964. 367 pp. (In Russian)

[7] Pechenkin, A.A. Obosnovanie nauchnoy teorii: klassika and sovremennost [Foundations of Scientific Theories: Classics and Modern Times]. Moscow: Nauka, 1991. 184 pp. (In Russian)

[8] Schroedinger E. Izbrannye trudy po kvantovoi mehanike [Selected works in Quantum Mechanics]. Moscow: Mir, 1980, 420 pp. (In Russian)

[9] Birkhoff, G., von Neumann, J. "The Logic of Quantum Mechanics", Annals of Mathematics, 1936, Vol. 37, No. 4, pp. 823-843.

[10] van Fraassen, B. "The labyrinth of quantum logics", in: Boston Studies in the Philosophy of Science, ed. by R.S. Cohen and M.W. Vastovsky. Dordrehct: Reidel Publishing Company, 1972, pp. 224-254.

[11] Hanson, N.R. The concepr of positron. Cambridge: Cambr. Univ. Press, 1965. $236 \mathrm{pp}$.

[12] Hooker, C. The logico-algebraic approach to Quantum Mechanics. Dordrehct: Reidel Publishing Company, 1975. 469 pp.

[13] Jammer, M. The conceptual development of quantum mechanics. N.Y.: Wiley, 1966. 308 pp.

[14] Redei, M. "John von Neumann on mathematical and axiomatic physics", in: The role of mathematics in physical sciences, ed by G. Boniolo et. al. Netherlands: Springer, 2005, pp. 43-52.

[15] Redei, M. "Von Neumann's concept of quantum logic and quantum probability", in: John von Neumann and the Foundations of Quantum Physics, ed. by M. Redei, M. Stotzner. Netherlands: Springer, 2001, $464 \mathrm{pp}$.

[16] Wilce, A. "Quantum logic and probability theory", Stanford encyclopedia of philosophy. 2002. Substantive revision - 2017. [https://plato.stanford.edu/ entries/qt-quantlog/, accessed on 30.08.2017]. 